\title{
Deglutição e fala de pacientes submetidos à glossectomia devido ao câncer de língua: relato de casos
}

\author{
Swallowing and speech of patients submitted to glossectomy due to \\ tongue cancer: cases report
}

Manuela Lunardi Burtet ${ }^{1}$ (D), Liliane Janete Grando² (ㄱ, Cláudia Tiemi Mituuti ${ }^{1}$

\section{RESUMO}

Este estudo teve como objetivo correlacionar o grau de comprometimento da deglutição e da fala de pacientes submetidos à glossectomia devido ao câncer de língua. Trata-se de um estudo de casos, de caráter transversal observacional, realizado em sete pacientes, com idade acima de 18 anos, que realizaram glossectomia como tratamento do câncer de língua. Foram excluídos da pesquisa pacientes que possuíam doenças neurológicas, ou que realizaram outras cirurgias de cabeça e pescoço, não relacionadas ao câncer de língua. Foi realizada avaliação clínica da deglutição, por meio do protocolo Avaliação de Segurança da Deglutição (ASED) e classificação da ingestão oral, conforme a Escala Funcional de Ingestão por Via Oral (Functional Oral Intake Scale - FOIS) A avaliação da fala se deu por meio do protocolo MBGR (Marchesan, Berrentin-Felix, Genaro, Rehder). Observou-se que as maiores alterações na deglutição ocorreram em fase oral da deglutição, havendo necessidade de modificações na alimentação, impactando o nível de ingestão oral. As alterações de fala mais encontradas foram distorção nos fonemas linguoalveolares /f/ e/1/e linguopalatais /s/, /z/, / //,/3/e imprecisão articulatória. Houve correlação entre as alterações de deglutição e o nível de ingestão oral e as alterações de fala, demonstrando que, quanto maiores as modificações presentes na alimentação, maiores as alterações de fala encontradas nos indivíduos do presente trabalho.

Palavras-chave: Fonoaudiologia; Transtornos da deglutição; Neoplasias bucais; Glossectomia; Transtornos da fala

\begin{abstract}
The present study aimed to correlate the swallowing and speech impairment level of patients submitted to glossectomy due to tongue cancer. This is a cross-sectional observational case study carried out with seven patients, over 18 years of age, who underwent glossectomy as a treatment for tongue cancer. Patients who had neurological diseases or who had undergone other head and neck surgeries not related to tongue cancer were excluded from the study. Swallowing clinical evaluation was performed using the ASED (Avaliação de Segurança da Deglutição) protocol and classification of oral intake according to the FOIS scale. Speech assessment was performed using the MBGR (Marchesan, Berrentin-Felix, Genaro, Rehder) protocol. It was observed that the major changes in swallowing occurred in the oral phase of swallowing, requiring changes in feeding practices with an impact on the level of oral intake. The following speech changes were mostly found: distortion in lingual-alveolar phonemes $/ \mathrm{s} /$ and $/ 1 /$ and lingual-palatals $/ \mathrm{s} /, / \mathrm{z} /, / \mathrm{J} /, / 3 /$ and articulatory inaccuracy. A correlation was found between swallowing changes and oral food intake level and speech changes, demonstrating that the greater the feeding changes, the greater the speech changes found in the individuals of the present investigation.
\end{abstract}

Keywords: Speech therapy; Deglutition disorders; Mouth neoplasms; Glossectomy; Speech disorders

Trabalho realizado na Universidade Federal de Santa Catariana - UFSC - Florianópolis (SC), Brasil.

${ }^{1}$ Departamento de Fonoaudiologia, Universidade Federal de Santa Catariana - UFSC - Florianópolis (SC), Brasil.

${ }^{2}$ Departamento de Patologia, Universidade Federal de Santa Catariana - UFSC - Florianópolis (SC), Brasil.

Conflito de interesses: Não.

Contribuição dos autores: MLB revisão bibliográfica, coleta e análise dos dados, redação final do artigo; LJG idealização e delineamento metodológico do estudo; CTM idealização e delineamento metodológico do estudo, revisão bibliográfica, análise dos dados, redação final do artigo.

Financiamento: Nada a declarar.

Autor correspondente: Cláudia Tiemi Mituuti. E-mail: claudia.mituuti@ufsc.br

Recebido: Maio 02, 2019; Aceito: Fevereiro 25, 2020 


\section{INTRODUÇÃO}

O câncer de cavidade oral abrange tumores localizados na língua bucal, assoalho da boca, gengiva, mucosa jugal e palato duro. Segundo o Instituto Nacional do Câncer (INCA), a estimativa de novos casos de câncer de cavidade oral, para 2018, foi de 11.200 novos casos para homens e 3.500 para mulheres. Os principais fatores de risco estão associados ao tabagismo e ao etilismo, sendo que, quando combinados, aumentam a chance de adquirir a doença. Má alimentação, genética, má higiene oral e o vírus HPV também estão entre os fatores de risco ${ }^{(1,2)}$.

Para escolha da terapêutica, é necessário determinar o estadiamento do tumor, cujo objetivo é avaliar o grau de disseminação da doença. Os tratamentos mais utilizados são a cirurgia, a radioterapia (RTX) e a quimioterapia (QTX). Considerando as funções da cavidade oral, como a mastigação, a deglutição e a fala, a escolha do tratamento utilizado deve considerar vários fatores, para garantir a qualidade de vida, assim como o favorecimento da sobrevivência ${ }^{(3)}$.

Os efeitos na fala e na deglutição dos indivíduos que realizam cirurgia são heterogêneos e variam de acordo com o local da ressecção e do tipo de reconstrução. A literatura demonstra que, em indivíduos submetidos à glossectomia, há aumento do tempo de trânsito oral, na deglutição, e da quantidade de resíduos em cavidade oral, com piora na eficiência da deglutição e redução da porcentagem de pacientes habilitados a ingerir alimentos pastosos e sólidos após a cirurgia. Além disso, há piora da inteligibilidade de fala e significante redução na produção correta dos fonemas após a cirurgia, sem recuperação ao longo do tempo ${ }^{(4)}$.

Diante das sequelas da cirurgia oncológica para o tratamento do câncer de língua, as alterações da fala podem estar relacionadas ao comprometimento da deglutição. Portanto, o objetivo do presente estudo foi descrever as alterações de deglutição e fala de pacientes submetidos à glossectomia devido ao câncer de língua, além de correlacionar o nível de ingestão oral e as alterações da deglutição com as alterações da fala destes indivíduos.

\section{APRESENTAÇÃO DO CASO CLÍNICO}

Trata-se de um estudo transversal observacional, aprovado pelo Comitê de Ética em Pesquisas com Seres Humanos - CEPSH (Número do Parecer: 1.939.138) da Universidade Federal de Santa Catarina. Todos os pacientes foram informados sobre os objetivos da pesquisa e assinaram o Termo de Consentimento
Livre e Esclarecido (TCLE) para a realização dos procedimentos e utilização dos dados.

Foram incluídos no estudo pacientes adultos, acima de 18 anos e idosos de ambos os gêneros, que realizaram glossectomia como tratamento do câncer de língua, há, no mínimo 45 dias da cirurgia e, quando tratados com quimioterapia (QTX) e radioterapia (RTX), há 45 dias após a última sessão ${ }^{(5)}$. Os critérios de exclusão foram pacientes que possuíam doenças neurológicas ou que realizaram outras cirurgias de cabeça e pescoço, não relacionadas ao câncer de língua.

Enquadraram-se nos critérios de inclusão sete pacientes, com idades entre 35 e 79 anos, média de 56,28 anos ( $\mathrm{DP}=+-13,37$ anos), todos do gênero masculino. Seis deles referiram ser ex-tabagistas. A quantidade de cigarros por dia variou de nove a 60 e o tempo de exposição ao vício variou entre dez e 54 anos.

Quanto à localização do tumor, todos os pacientes realizaram ressecção em bordo de língua. Os dados referentes à idade, ao hábito, tipo histológico e localização do tumor estão apresentados na Tabela 1.

Em relação ao tratamento, todos realizaram glossectomia parcial. Além da cirurgia como tratamento do câncer, quatro pacientes realizaram RTX, dois pacientes realizaram QTX e um paciente não realizou tratamento adjuvante. O número de sessões de RTX variou de 28 a 35 sessões. A dose de radiação variou de 60 a 67 Gys para três pacientes e, para os demais, estes dados não estavam apresentados no prontuário. A realização de esvaziamento cervical se deu em cinco pacientes (P1, P3, P4, P5 e P6). Seis (P1, P2, P4, P5, P6 e P7) dos sete pacientes haviam realizado terapia fonoaudiológica anteriormente.

Todos os indivíduos realizaram as avaliações fonoaudiológicas, no mínimo, 45 dias após o tratamento médico. A coleta de dados foi feita por meio da avaliação clínica da deglutição e da fala, enquanto que os dados de caracterização dos pacientes (cirurgia, tratamento com RTX e QTX, estadiamento do tumor na época do tratamento e localização) foram obtidos do prontuário.

Para avaliação da deglutição foi realizada a avaliação clínica, por meio do protocolo de Avaliação de Segurança da Deglutição (ASED) ${ }^{(6)}$, utilizado para avaliação estrutural, vocal e funcional da deglutição, em que foram utilizadas as consistências líquida, pudim e sólida, para verificar os sinais clínicos de penetração/aspiração. Quando presentes, as alterações de fase oral e fase faríngea foram pontuadas para análise estatística. A pontuação final foi determinada pela somatória das alterações presentes em todas as consistências.

Foi realizada a investigação da ingestão oral, por meio do recordatório alimentar de 24 horas. Os pacientes foram classificados conforme os níveis da Escala Funcional de Ingestão por Via Oral (Functional Oral Intake Scale - FOIS) ${ }^{(7)}$, considerando as

Tabela 1. Descrição dos dados individuais quanto ao uso de cigarro, esvaziamento cervical e quanto às informações sobre o tumor

\begin{tabular}{cccccc}
\hline Nome & $\begin{array}{c}\text { Idade } \\
\text { (anos) }\end{array}$ & Cigarros / dia & $\begin{array}{c}\text { Tipo } \\
\text { histológico }\end{array}$ & Localização do tumor & Esvaziamento cervical \\
\hline P1 & 53 & 15 & CEC & Bordo anterior e assoalho da boca & Sim (à esquerda) \\
P2 & 51 & 60 & CEC & Bordo de língua & Não \\
P3 & 79 & 2 cachimbos & CEC & Bordo anterior de língua & Sim (N/I) \\
P4 & 62 & 3 de palha & CEC & Bordo lateral de língua & Sim (à esquerda) \\
P5 & 35 & 0 & CEC & Bordo lateral de língua & Sim (à esquerda) \\
P6 & 53 & 8 & CEC & Bordo anterior de língua & Sim (N/I) \\
P7 & 61 & 60 & CEC & Bordo lateral de língua & Não \\
\hline
\end{tabular}


características da dieta, baseadas nas propriedades e textura dos alimentos. Os níveis variam de 1 a 7, sendo o nível 1 referente a nada por via oral e o nível 7 referente aos pacientes com dieta oral total sem quaisquer restrições alimentares.

A avaliação da fala foi feita por meio do protocolo MBGR (Marchesan, Berrentin-Felix, Genaro, Rehder) ${ }^{(8)}$, que possibilita avaliar as condições anatômicas e funcionais do sistema estomatognático. A partir deste protocolo, foi realizada apenas a avaliação referente à fala, que consistiu em cinco provas: prova 1 : fala automática, com contagem de zero a 20 , dias da semana e meses do ano; prova 2: nomeação de 50 figuras contidas no protocolo; prova 3: coordenação motora da fala, solicitando que o paciente realizasse a emissão rápida e repetida dos sons [pa],[ta],[ka],[pataka]; prova 4: fala espontânea, utilizando perguntas, como "diga seu nome e quantos anos você tem", "diga o que você faz" (estuda, trabalha); prova 5: repetição de 34 palavras, balanceadas foneticamente, contidas no protocolo. A pontuação das provas individuais foi realizada pela somatória de alterações presentes. A somatória das alterações presentes em todas as provas foi feita para determinar a pontuação final.

Todas as provas foram gravadas em vídeo, com câmera digital da marca CANON, modelo SD1200 IS, apoiada sobre tripé de mesa, a um metro de distância do paciente, com enquadramento entre os ombros e cabeça, para análise dos aspectos fonéticos.

Sendo assim, os dados obtidos por meio das avaliações da deglutição e da fala foram tabulados em banco de dados específico para aplicação de teste estatístico pertinente. A correlação entre os resultados da avaliação clínica da deglutição, da FOIS e da fala foram analisados por meio do teste de correlação de Spearman, considerando valor de $p \leq 0,05$. A correlação entre a avaliação clínica da deglutição e avaliação da fala foi realizada levando em conta as alterações presentes em todas as consistências em fase oral, fase faríngea e na sua somatória.
$\mathrm{Na}$ avaliação funcional da deglutição, cinco pacientes tiveram alteração em algum momento da avaliação clínica. Na fase oral, quatro pacientes apresentaram alterações, prevalecendo o resíduo em cavidade oral. Na fase faríngea, dois pacientes apresentaram tosse e pigarro na consistência líquida, sendo que um apresentou elevação laríngea não eficiente e ausculta cervical positiva. Três pacientes apresentaram tosse e/ou pigarro na consistência pudim e nenhuma alteração foi observada quando ofertado sólido. Foi verificado que os pacientes 1 e 2 não faziam ingestão de sólidos no momento da avaliação (Tabela 2).

Em relação ao nível de ingestão oral dos pacientes, três tiveram classificações nível 5, um paciente, nível 6 e três pacientes, nível 7 (Figura 1).

$\mathrm{Na}$ avaliação da fala, a maioria dos indivíduos apresentou alteração, sendo que as mais encontradas foram distorção nos fonemas líquidos / / /, /l/, nos fricativos /s/, /z/, / / / e/z/ e imprecisão articulatória. Os dados individuais dos fonemas alterados e a somatória das alterações para a pontuação final se encontram na Tabela 3.

A partir dos resultados, foi realizada a correlação entre os dados de alterações de deglutição e da pontuação final das provas de fala, por meio do teste de correlação de Spearman, em que foi verificada correlação entre as alterações de fase oral $(\mathrm{p}=0,055)$, fase faríngea $(\mathrm{p}=0,039)$ e a somatória das alterações encontradas nas fases $(0,028)$, considerando todas as consistências testadas, demonstrando que, quanto maior a presença de alterações na deglutição, maior a pontuação nas provas de fala.

A partir da análise de correlação entre a FOIS e a pontuação da fala, foi verificada correlação negativa com valor de $\mathrm{p}=0,039$, demonstrando que quanto maiores as alterações de fala, menor a classificação no nível de ingestão oral. Os dados de correlação estão descritos na Tabela 4.

Tabela 2. Resultados individuais das alterações presentes em fase oral e faríngea da deglutição, a partir da avaliação funcional da deglutição por meio do protocolo de Avaliação de Segurança da Deglutição, de acordo com as consistências testadas

\begin{tabular}{|c|c|c|c|c|c|c|c|c|c|c|c|c|c|c|c|c|c|c|c|c|c|}
\hline \multicolumn{22}{|c|}{ ASED } \\
\hline & $\mathrm{P} 1$ & $\mathrm{P} 2$ & P3 & P4 & P5 & P6 & P7 & $\mathrm{P} 1$ & $\mathrm{P} 2$ & P3 & $\mathrm{P} 4$ & P5 & P6 & P7 & $\mathrm{P} 1$ & $\mathrm{P} 2$ & P3 & $\mathrm{P} 4$ & P5 & P6 & P7 \\
\hline & \multicolumn{7}{|c|}{ Liquido } & \multicolumn{7}{|c|}{ Pudim } & \multicolumn{7}{|c|}{ Sólido } \\
\hline \multicolumn{22}{|c|}{ FASE ORAL } \\
\hline Captação & $E$ & $E$ & $E$ & $E$ & $E$ & $E$ & $E$ & $E$ & $E$ & $E$ & $E$ & $E$ & $E$ & $E$ & NR & NR & $E$ & $E$ & $E$ & $E$ & $E$ \\
\hline Vedamento & $E$ & $E$ & E & $E$ & $E$ & $E$ & $E$ & $E$ & $E$ & E & $E$ & $E$ & $E$ & $E$ & NR & NR & $E$ & $E$ & $E$ & $E$ & $E$ \\
\hline Preparo & $E$ & $E$ & $E$ & $E$ & $E$ & $E$ & $E$ & $E$ & $E$ & $E$ & $E$ & $E$ & $E$ & $E$ & NR & NR & $E$ & $E$ & $E$ & $E$ & $E$ \\
\hline Coord. & $E$ & $\mathrm{NE}$ & $E$ & $E$ & $E$ & $E$ & $E$ & $E$ & $\mathrm{NE}$ & $E$ & $E$ & $E$ & $E$ & $\mathrm{E}$ & NR & NR & $E$ & $E$ & $E$ & $E$ & $E$ \\
\hline Res.em CO & $A$ & $A$ & $A$ & $A$ & $A$ & $A$ & $\mathrm{~A}$ & $A$ & $A$ & $A$ & $A$ & A & $A$ & $\mathrm{~A}$ & NR & NR & $P$ & $P$ & $A$ & $P$ & A \\
\hline \multicolumn{22}{|c|}{ FASE FARÍNGEA } \\
\hline $\begin{array}{l}\text { Elevação } \\
\text { laringea }\end{array}$ & NE & E & E & E & E & E & E & $E$ & $\mathbf{E}$ & E & $\mathbf{E}$ & E & E & $\mathbf{E}$ & NR & NR & $\mathbf{E}$ & E & E & $E$ & E \\
\hline Desconforto & $A$ & $A$ & $A$ & $A$ & $A$ & $A$ & $A$ & $A$ & A & A & $A$ & A & $A$ & A & NR & NR & $A$ & $A$ & $A$ & $A$ & A \\
\hline Ausculta & + & - & - & - & - & - & - & - & - & - & - & - & - & - & NR & NR & - & - & - & - & - \\
\hline
\end{tabular}

Legenda: ASED = avaliação de segurança da deglutição; $\mathrm{P}(1,2,3,4,5,6,7)=$ paciente; TTO = tempo de trânsito oral; Coord. = coordenação entre as fases oral e faríngea; Res. em CO = resíduo em cavidade oral; $\mathrm{E}=$ eficiente; $\mathrm{NE}$ = não eficiente; $\mathrm{A}=$ ausente; $\mathrm{P}=$ presente; $\mathrm{NR}=$ não realizado; - = ausculta cervical negativa; $+=$ ausculta cervical positiva 


\section{ESCALA FOIS}

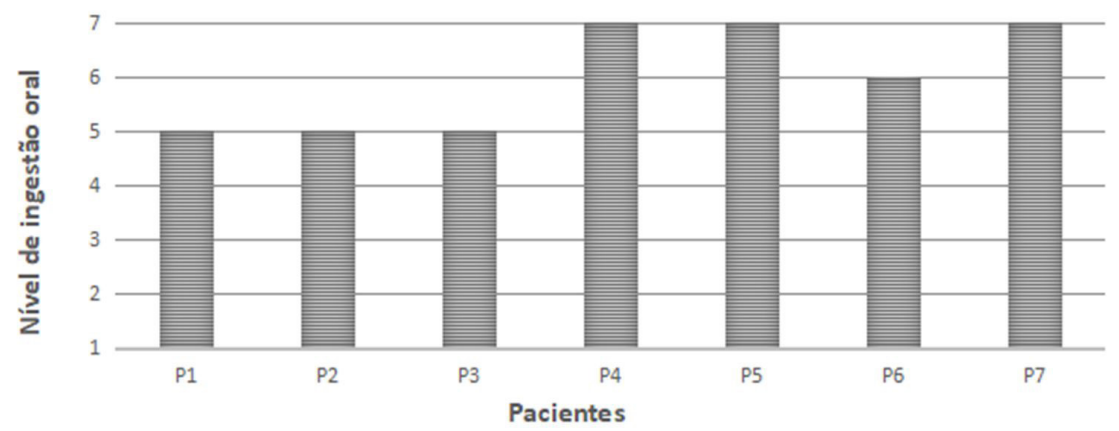

Figura 1. Resultados individuais da classificação do nível de ingestão oral dos pacientes por meio da Functional Oral Intake Scale Legenda: FOIS = Functional Oral Intake Scale

Tabela 3. Resultados individuais da classificação da alteração da fala, dos fonemas alterados e pontuação final da fala, avaliados por meio da avaliação miofuncional orofacial - protocolo MBGR

\begin{tabular}{cccc}
\hline Paciente & Classificação da fala & Fones alterados & Pontuação final da fala \\
\hline P1 & Alterada & $/ \mathrm{s} /$ & 10 pontos \\
P2 & Alterada & $/ \mathrm{s} /, / / / / \mathrm{k} /, / \mathrm{g} /$ & 18 pontos \\
P3 & Alterada & $/ \mathrm{s} /, / / /, / \mathrm{s} /, / \mathrm{s} /$ & 11 pontos \\
P4 & Adequada & Nenhum & 0 pontos \\
P5 & Adequada & Nenhum & 0 pontos \\
P6 & Alterada & $/ \mathrm{s} /, / / /, / \mathrm{s} /, / \mathrm{z} /, / 3 /$ & 14 pontos \\
P7 & Alterada & $/ \mathrm{s} /, / \mathrm{z} /$ & 8 pontos \\
\hline
\end{tabular}

Legenda: MBGR = Marchesan, Berrentin-Felix, Genaro, Rehder

Tabela 4. Valores de $r$ e de $p$ obtidos por meio do teste de correlação de Spearman para análise das variáveis das alterações presentes nas provas de deglutição e da pontuação final da fala, considerada correlação significativa quando o valor de $p$ foi $\leq a 0,05$

\begin{tabular}{cccc}
\hline Prova de deglutição & Prova de fala & Valor de $\mathbf{r}$ & Valor de $\mathbf{p}$ \\
\hline Fase oral & Pontuação final & 0,458 & 0,055 \\
Fase faríngea & & 0,489 & 0,039 \\
Somatória das alterações & & 0,518 & 0,028 \\
FOIS & & $-0,778$ & 0,039 \\
\hline
\end{tabular}

Legenda: FOIS = Functional Oral Intake Scale; $r=$ coeficiente de correlação; $p=p$-valor

\section{DISCUSSÃO}

Este estudo teve como objetivo descrever as alterações de deglutição e fala de pacientes submetidos à glossectomia devido ao câncer de língua. Os achados quanto ao tipo histológico, prevalência entre homens e mulheres e o sítio acometido pelo câncer concordam com os dados da literatura brasileira, considerando o tipo histológico, gênero, idade e hábitos. A literatura demonstrou que o tipo histológico mais encontrado no câncer de cavidade oral é o carcinoma epidermoide (CEC), responsável por mais de $90 \%$ dos casos, sendo os sítios mais acometidos a língua e o assoalho da boca. Ainda, a doença atinge, principalmente, o sexo masculino, na faixa etária acima de 50 e, entre os principais fatores de risco estão o tabagismo e o etilismo, além de fatores genéticos, condições socioeconômicas e o vírus HPV ${ }^{(1,2)}$.

Todos os indivíduos do presente estudo realizaram cirurgia e a maioria realizou esvaziamento cervical e RTX como tratamento médico para o tumor. A cirurgia tem sido descrita como a modalidade terapêutica utilizada para o controle local da doença. A glossectomia pode causar problemas funcionais por desempenhar papel fundamental na deglutição e na fala. O comprometimento dessas funções está atrelado à extensão e localização da ressecção, à função da língua remanescente, ao estadiamento do tumor e ao comprometimento dano ganglionar ${ }^{(9)}$.

Considerando que todos os casos realizaram a glossectomia como modalidade de tratamento, a disfagia ocorre pela extensa perda de tecido, limitação da excursão do tecido remanescente, diminuição da mobilidade e sensibilidade da língua, do palato mole e da faringe ${ }^{(10)}$. Um estudo realizado por Son et al. ${ }^{(10)}$ em 133 pacientes que realizaram glossectomia, verificou que as alterações significantes após a cirurgia foram controle inadequado de língua, da mastigação e tempo de trânsito oral aumentado, além de aspiração, resíduos na faringe e elevação da laringe inadequada, visualizados por meio da videofluoroscopia da deglutição. Estes achados concordam com os dados do presente estudo, sendo que a maior frequência de alterações encontradas na avaliação clínica da deglutição foi em fase oral, com maior incidência de resíduo em cavidade oral após a deglutição.

A gravidade da disfagia em pacientes submetidos à glossectomia dependerá da extensão da ressecção, da mobilidade da estrutura 
remanescente, do tipo de reconstrução, do comprometimento de estruturas adjacentes e da motivação e adaptação do paciente com o resultado da cirurgia ${ }^{(11)}$. Em um estudo realizado por Huang et al. ${ }^{(12)}$ em 112 pacientes que realizaram glossectomia de diferentes extensões cirúrgicas, observou-se que os pacientes que foram submetidos à ressecção de língua maior que 50\% tiveram maior risco de aspiração na deglutição, quando comparados com os que realizaram ressecções menores, ou seja, a glossectomia parcial influenciou em menor grau a deglutição dos pacientes. Este achado também concorda com os dados do presente estudo, visto que todos os pacientes realizaram glossectomia parcial, com ressecção da língua menor que 50\% e somente dois pacientes demonstraram sinais clínicos de penetração/aspiração durante a avaliação clínica da deglutição.

A limitação da ingestão oral resultante da dificuldade na propulsão do bolo alimentar é comum em pacientes de pós-operatório de câncer de cabeça e pescoço, como um sinal de disfagia orofaríngea grave, mesmo sem sinais de aspiração ${ }^{(13)}$. No estudo de Hey et al. ${ }^{(13)}$, com 80 pacientes com câncer de cavidade oral, orofaringe e hipofaringe, foi constatado que $56,3 \%$ apresentaram nível de ingestão oral menor ou igual a 4. Os resultados do presente trabalho também demonstraram que a maioria $(n=4)$ apresentou alguma limitação na ingestão oral, porém nenhum deles abaixo do nível 4. Os resultados encontrados podem ter sido divergentes devido ao fato de a avaliação da deglutição dos pacientes desta pesquisa ter ocorrido em pós-operatório tardio (média de 3,6 anos), período no qual já realizam suas próprias adaptações para a deglutição, como alternância de consistências durante a alimentação e restrição dos alimentos sólidos.

No presente estudo, a maioria dos indivíduos apresentou alteração na fala, sendo que as mais frequentes foram distorção dos fonemas linguoalveolares (/r/ e/1/), linguopalatais $(/ \mathrm{J} /, / 3 / \mathrm{e} / \mathrm{K} /$ ) e imprecisão articulatória. A localização da ressecção está relacionada com a alteração desses fonemas, pois a maioria dos pacientes realizou ressecção em bordo lateral de língua, região necessária para a produção dos fonemas linguopalatais $(/ \mathrm{J} / \mathrm{/} / \mathrm{Z} / \mathrm{e} / \mathrm{K} /)$. Estes achados estão de acordo com os resultados encontrados por Jaya et al. ${ }^{(14)}$, que verificaram que, após a cirurgia em indivíduos com câncer de língua, os erros mais frequentes foram substituição, distorção e omissão de fonemas bilabiais, linguoalveolares e linguopalatais, além de inteligibilidade de fala comprometida.

Os resultados do presente estudo demonstraram que, quanto maior a alteração na deglutição e no nível de ingestão oral, maior a alteração na fala. Isto se dá pelo fato de a língua ser uma estrutura fundamental, tanto na deglutição, como na fala, sendo responsável pelo controle e ejeção do bolo alimentar, lateralização durante a mastigação, proteção das vias aéreas, além de ser um dos principais articuladores da fala ${ }^{(15)}$. No estudo realizado por Hey et al. ${ }^{(13)}$, verificou-se que a disglossia, voz molhada, mobilidade e força de língua, observadas durante a avaliação clínica, tiveram correlação significativa com a limitação da ingestão oral e, exceto a disglossia, com a aspiração, demonstrando a importância dos parâmetros clínicos de funções orais e laríngeas não relacionados à deglutição como preditores de limitação da ingestão oral e de aspiração.

Embora o presente estudo tenha apresentado resultados significantes na correlação de seus achados, o número reduzido de pacientes foi uma limitação. Além disso, a avaliação instrumental da deglutição poderia auxiliar na análise objetiva dos aspectos da sua biodinâmica, relacionados ao tempo, coordenação e amplitude do movimento e suas correlações com os achados da biodinâmica da fala.

\section{COMENTÁRIOS FINAIS}

Os indivíduos do presente estudo, submetidos à glossectomia decorrente do câncer de língua, apresentaram maiores alterações em fase oral da deglutição e modificações na alimentação, com impacto no nível de ingestão oral. As alterações de fala mais presentes foram distorção e imprecisão articulatória. Houve correlação entre as alterações de deglutição e o nível de ingestão oral e as alterações de fala, demonstrando que, quanto maiores as alterações presentes na alimentação, maiores as alterações de fala encontradas.

\section{REFERÊNCIAS}

1. Bezerra NVF, Leite KLF, Medeiros MMD, Martins ML, Cardoso AMR, Alves PM, et al. Impact of the anatomical location, alcoholism and smoking on the prevalence of advanced oral cancer in Brazil. Med Oral Patol Oral Cir Bucal. 2018 Maio;23(3):e295-301. http://dx.doi. org/10.4317/medoral.22318. PMid:29680854.

2. INCA: Instituto Nacional de Câncer José Alencar Gomes da Silva. Estimativa 2018: incidência de câncer no Brasil. Rio de Janeiro: INCA; 2017.

3. Omura K. Current status of oral cancer treatment strategies: surgical treatments for oral squamous cell carcinoma. Int J Clin Oncol. 2014 Abr;19(3):423-30. http://dx.doi.org/10.1007/s10147-014-0689-z. PMid:24682763.

4. Pauloski BR, Logemann JA, Rademaker AW, McConnel FMS, Heiser MA, Cardinale S, et al. Speech and swallowing function after anterior tongue and floor of mouth resection with distal flap reconstruction. $\mathrm{J}$ Speech Hear Res. 1993 Abr;36(2):267-76. http://dx.doi.org/10.1044/ jshr.3602.267. PMid:8487519.

5. Barbon C, Hope A, Steele C. Radiation 101: a guide for speechlanguage pathologists. Perspect ASHA Spec Interest Groups. 2017 Abr;2(13):63-72. http://dx.doi.org/10.1044/persp2.SIG13.63.

6. Furkim AM, Duarte ST, Sória FS, Sampaio RS, Nunes MCN, Wolff GS, et al. Evaluación clínica de las disfagias orofaríngeas. In: Susanibar F, Marchesan I, Parra D, Dioses A, editores. Tratado de evaluación de motricidad orofacial y áreas afines. Madrid: EOS; 2014. p. 233-46.

7. Crary MA, Mann GDC, Groher ME. Initial psychometric assessment of a functional oral intake scale for dysphagia in stroke patients. Arch Phys Med Rehabil. 2005 Ago;86(8):1516-20. http://dx.doi.org/10.1016/j. apmr.2004.11.049. PMid:16084801.

8. Genaro KF, Berretin-Felix G, Rehder MIBC, Marchesan IQ. Protocolo miofuncional orofacial: protocolo MBGR. Rev CEFAC. 2009 AbrJun;11(2):237-55. http://dx.doi.org/10.1590/S1516-18462009000200009.

9. Ji YB, Cho YH, Song CM, Kim YH, Kim JT, Ahn HC, et al. Long-term functional outcomes after resection of tongue cancer: determining the optimal reconstruction method. Eur Arch Otorhinolaryngol. 2017 Jul;274(10):3751-6. http://dx.doi.org/10.1007/s00405-017-4683-8. PMid:28748261.

10. Son YR, Choi KH, Kim TG. Dysphagia in tongue cancer patients. Ann Rehabil Med. 2015 Abr;39(2):210-7. http://dx.doi.org/10.5535/ arm.2015.39.2.210. PMid:25932417.

11. Furia CLB, Carrara-de-Angelis C, Martins NMS, Barros APB, Carneiro B, Kowalski LP. Video fluoroscopic evaluation after glossectomy. Arch Otolaryngol Head Neck Surg. 2000 Mar;126(3):378-83. http:// dx.doi.org/10.1001/archotol.126.3.378. PMid:10722012. 
12. Huang ZS, Chen W, Huang ZQ, Yang ZH. Dysphagia in tongue cancer patients before and after surgery. J Oral Maxillofac Surg. 2016 Out;74(10):206772. http://dx.doi.org/10.1016/j.joms.2016.03.031. PMid:27126392.

13. Hey C, Lange BP, Aere C, Eberle S, Zaretsky Y, Sader R, et al. Predictability of oral and laryngopharyngeal function for aspiration and limitation of oral intake in patients after surgery for head and neck cancer. Anticancer Res. 2013 Out;33(8):3347-54. PMid:23898102.
14. Jaya V, Saravanan G, Ranganathan V, Gandhi A. Speech outcome in oral cancer patients - pre- and post-operative evaluation: a crosssectional study. Indian J Palliat Care. 2016 Out-Dez;22(4):499-503. http://dx.doi.org/10.4103/0973-1075.191858. PMid:27803574.

15. Vieira CA. Fonoterapia em glossectomia total. Rev Soc Bras Fonoaudiol. 2011 Dez;16(4):479-82. http://dx.doi.org/10.1590/S151680342011000400019 . 